\title{
LE TERS, OU COMMENT OBTENIR une signature chimique à l'échelle nanométrique
}

\author{
Patrick HSIA \\ Marc CHAIGNEAU \\ HORIBA France SAS, 59650 \\ Villeneuve d'Ascq, France \\ HORIBA France SAS, 91120 \\ Palaiseau, France \\ marc.chaigneau@horiba.com
}

\begin{abstract}
Le TERS pour tip enhanced raman scattering, est une technique de pointe (dans tous les sens du terme) basée sur l'amplification du signal Raman relative à la résonance de plasmons de surface. Cette technique non-destructive, qui ne nécessite pas de marquage particulier, permet de caractériser un échantillon à l'échelle nanométrique. Le TERS s'impose aujourd'hui pour sonder les propriétés physico-chimiques des nanomatériaux et participer ainsi au développement de nouvelles applications dans le domaine des nanotechnologies.
\end{abstract}

Afin d'avoir des valeurs de décalage qui ne dépendent pas de la longueur d'onde incidente du laser, ils sont exprimés en différence de nombre d'onde par rapport à la longueur d'onde incidente $\lambda_{\text {inc }}$.

Raman shift $\left(\mathrm{cm}^{-1}\right)=10^{7}\left(\frac{1}{\lambda_{\text {inc }}}-\frac{1}{\lambda}\right)$ avec $\lambda$ et $\lambda_{\text {inc }}$ exprimés en nm.

Ainsi, un shift de $0 \mathrm{~cm}^{-1}$ correspond à un décalage nul. Majoritaires, ces photons sont les photons diffusés élastiquement. Cette diffusion est appelée diffusion Rayleigh

L'effet Raman est toutefois statistiquement rare : environ 1 photon diffusé sur 10000000 incidents est concerné par un décalage. Le défi technologique des instrumentistes

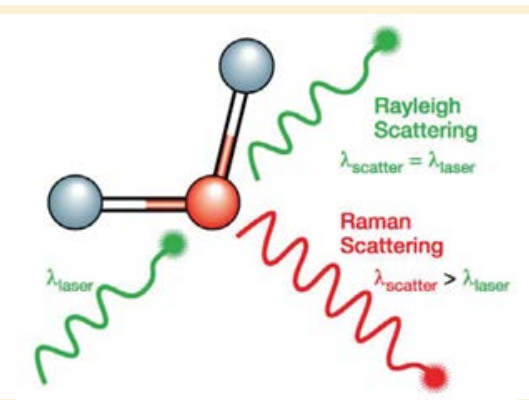

Figure 1. Principe de l'effet Raman. La diffusion de la lumière par une molécule peut être élastique (diffusion Rayleigh) ou non élastique (diffusion Raman). (Crédit : HORIBA Scientific)
« ramanistes » consiste ainsi à séparer le bon grain de l'ivraie: ne détecter que les photons concernés par un décalage et se débarrasser des photons issus de la diffusion Rayleigh et ce, avec la meilleure résolution spectrale.

\section{Sauts technologiques marquants}

À travers l'Histoire, plusieurs sauts technologiques ont participé à l'essor de la spectroscopie Raman:

- l'invention du laser a permis d'obtenir des sources monochromatiques fines et comportant un grand nombre de photons,

le développement de la microscopie a permis de focaliser un faisceau laser et de collecter la diffusion Rayleigh et la diffusion Raman à travers l'objectif du microscope,

la fabrication de composants optiques performants constituant la chaîne de mesure après collection du signal diffusé contribue également à obtenir un spectre avec un rapport signal sur bruit satisfaisant. On peut citer les filtres (edge, notch) avec des coupures de plus en plus nettes pour filtrer la diffusion Rayleigh, le réseau de diffraction qui va décomposer la lumière ou la caméra CCD qui va enregistrer le spectre. ou encore des contraintes mécaniques dans l'échantillon. 
Toutefois, comme la microscopie optique, la spectroscopie Raman se heurte à la barrière de la limite de diffraction : la résolution spatiale de la spectroscopie Raman est limitée par la taille du spot du faisceau focalisé et est donnée par le critère de Rayleigh.

\section{Le TERS, la nouvelle révolution}

Dans les années 80, les techniques de microscopie à sonde locale se développent. Au moyen d'une pointe ayant une extrémité de dimension nanométrique, il est possible de balayer la surface de l'échantillon en régulant la distance pointe - échantillon sur un signal de référence. Les résolutions spatiales de topographie obtenues sont de l'ordre de grandeur de la dimension de la pointe, ce qui ouvrit à l'époque l'opportunité de l'étude de nano-objets.

\section{La pointe, l'élément au coeur du TERS}

En combinant la spectroscopie Raman et les microscopies à sonde locale, il est possible de profiter des avantages de chacune des techniques. Ainsi, une nouvelle technique apparaît : le TERS.

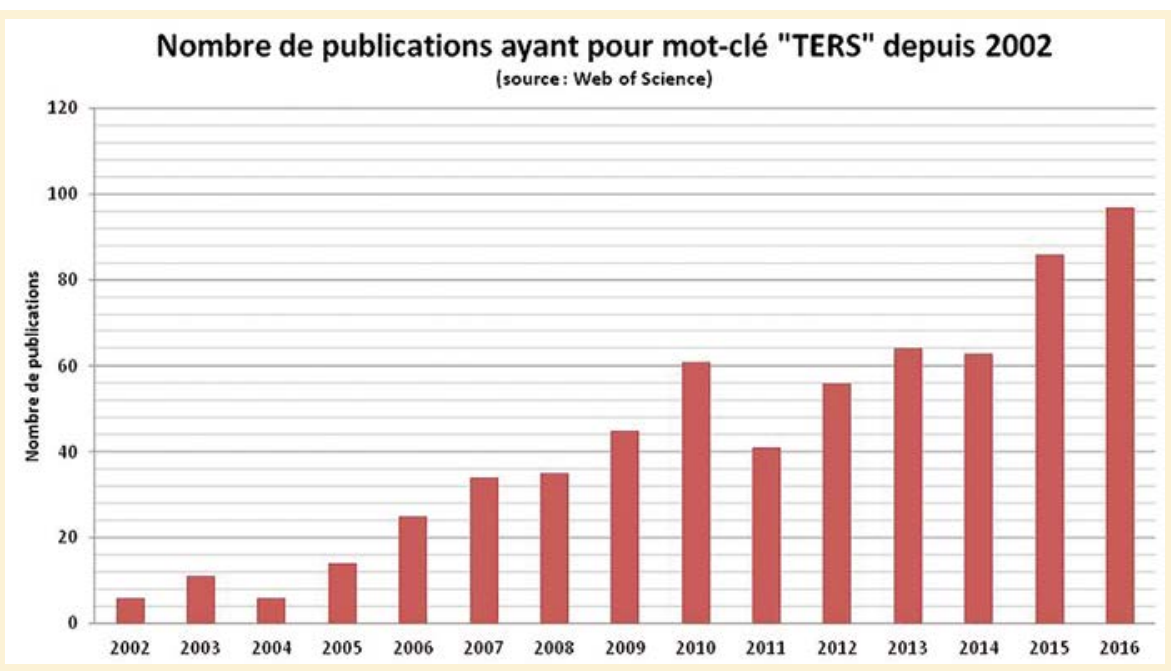

Figure 2. Nombre de publications ayant «TERS » comme l'un des mots-clefs. (Source: Web of Science)

La pointe y joue un double rôle.

- Amplifier l'excitation laser ainsi que la diffusion Raman (intrinsèquement faible) par le biais d'effets d'antenne et de résonance de plasmons combinés. En effet, la pointe est le siège d'effets d'amplification du champ électromagnétique, conséquence du cumul d'effets de résonance et d'effets géométriques. L'amplification résultante dépendra d'ailleurs grandement de la géométrie de la pointe. Les effets de résonance plasmonique sont principalement liés à la longueur d'onde d'excitation et à la géométrie de la sonde tandis que les effets géométriques sont assez dépendants de la polarisation de l'excitation.

Localiser l'information chimique donnée par la spectroscopie Raman à l'échelle de la dizaine de nanomètres. L'extrémité de la pointe agit comme une nanosource secondaire de lumière qui est capable de confiner l'amplification du champ électrique. C'est ce confinement nanométrique qui permet de s'affranchir de la limite de diffraction.

\begin{tabular}{|l|l|}
\hline EXCITATION \\
(ET COLLECTION) PAR...
\end{tabular}




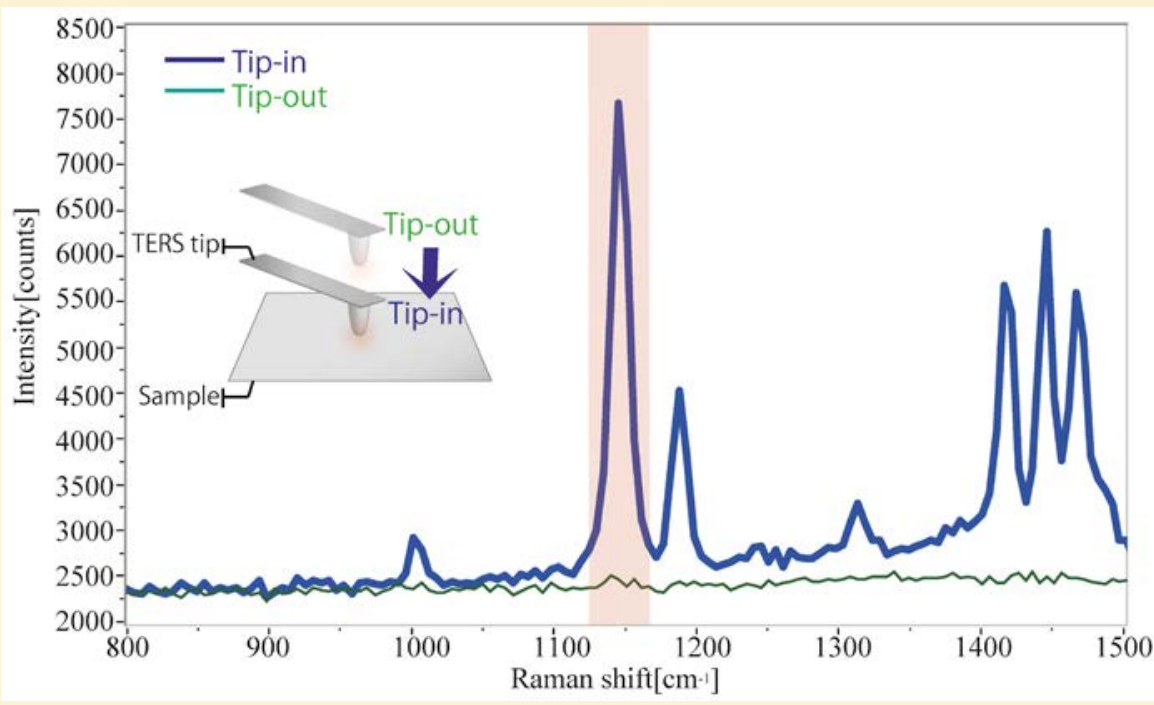

Figure 3. Spectre TERS d'une monocouche de molécules d'azobenzène greffées sur substrat d'or. En approchant la pointe au voisinage de la molécule, les pics Raman de l'azobenzène se dévoilent. (Crédit : HORIBA Scientific)

La pointe est donc le paramètre clef en TERS. La spectroscopie Raman bénéficie doublement de ces effets d'exaltation puisque la diffusion Raman est amplifiée au même titre que le champ incident. Différentes techniques de microscopie à sonde locale et donc différentes géométries de pointe (issue d'attaque électrochimique, montée sur cantilever ou sur diapason...) peuvent être utilisées. De plus, en considérant les différents modes d'utilisation de la microscopie à
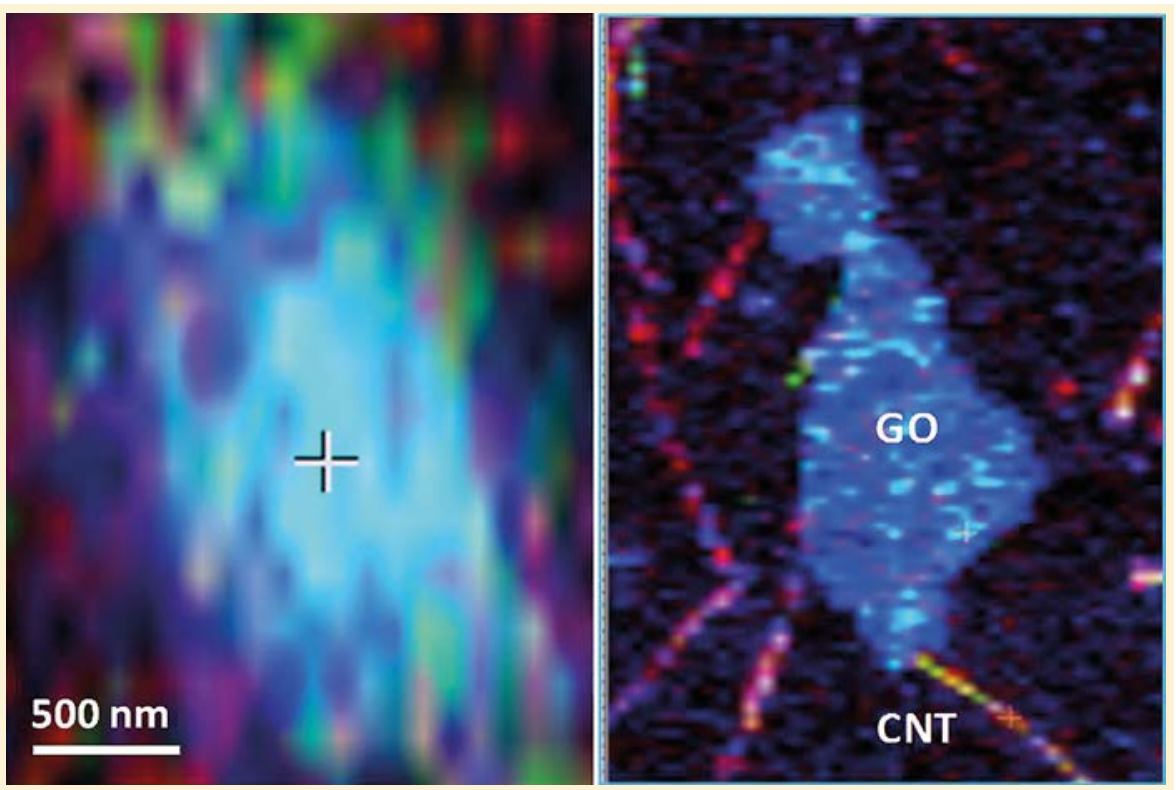

Figure 4. À gauche, cartographie $2 \mu \mathrm{m} \times 3 \mu \mathrm{m}$ en microscopie Raman confocale conventionnelle (puissance laser : $13 \mathrm{~mW}$, temps d'intégration $1 \mathrm{~s} / \mathrm{px}$ ). À droite, cartographie $2 \mu \mathrm{m} \times 3 \mu \mathrm{m}$ en utilisant le TERS (puissance laser : $130 \mu \mathrm{W}$, temps d'intégration $0,2 \mathrm{~s} / \mathrm{px}$ ). De nouveaux détails comme de l'oxyde de graphène $(\mathrm{GO})$ et des nanotubes de carbone (CNT) sont résolus à l'aide du TERS. (Crédit : HORIBA Scientific) force atomique (AFM), des informations chimiques locales peuvent être corrélées avec des informations topographiques ou encore des informations physiques (conductivité locale, potentiel de surface, capacitance, propriétés magnétiques et même mécaniques).

Les premiers articles scientifiques traitant de TERS remontent à l'année 2000. Depuis, le nombre de publications sur le TERS par année a une tendance à la hausse.

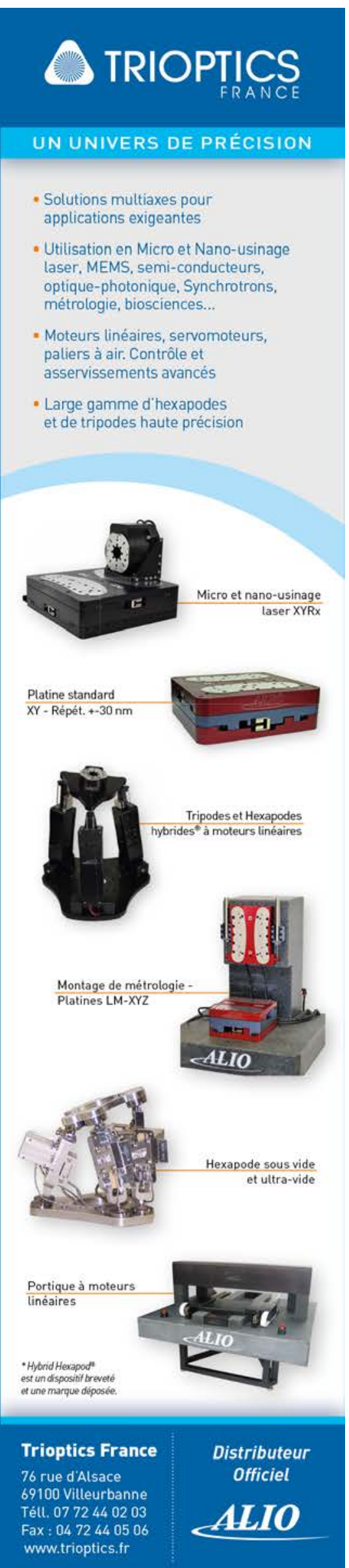




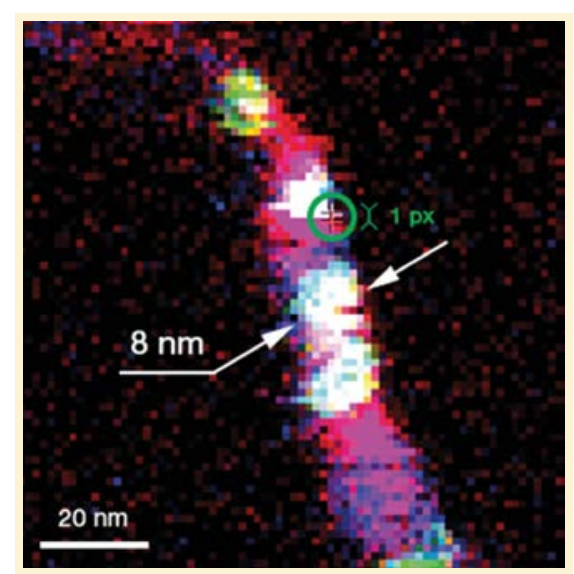

Figure 5. Cartographie TERS d'un nanotube de carbone unique. La résolution optique est estimée via l'analyse de la section de l'intensité des bandes TERS. (Crédit : HORIBA Scientific)

\section{Configurations expérimentales}

Le paramètre expérimental clef afin d'utiliser le TERS est l'alignement du laser sur l'extrémité de la pointe. Il existe plusieurs configurations pour y parvenir. Chacune possède ses avantages et ses inconvénients.

\section{Exemples de données que le TERS permet d'obtemin}

Une fois l'alignement optimisé, l'effet TERS sur le spectre Raman est observé quand l'échantillon et la pointe sont quasiment en contact puisque l'exaltation de champ est confinée (figure 3 ).

Il est également possible de répéter l'enregistrement d'un spectre TERS sur une succession de pixels afin de cartographier la surface d'un échantillon. Sur la figure 4, la résolution est meilleure sur l'image TERS, on distingue des nano-objets qui sont indiscernables sur l'image de microscopie confocale. De plus, la puissance laser ainsi que le temps d'acquisition ont été grandement diminués, respectivement d'un facteur 100 et d'un facteur 5 (et donc un temps total pour la cartographie moindre). Une résolution optique inférieure à $10 \mathrm{~nm}$ est aisément accessible en TERS sur les nanomatériaux carbonés (figure 5).

D'une manière générale, les spectres TERS ne sont reproductibles qu'à condition que les pointes utilisées pour les générer soient elles-mêmes reproductibles. Ainsi, la fabrication des pointes responsables de l'exaltation du signal Raman est un élément clef. La solution généralement adoptée consiste à déposer une couche mince d'or ou d'argent sur des pointes AFM en silicium ; des géométries de pointes plus ou moins exotiques existent également dans la littérature scientifique [1].

\section{De nouvelles applications que le TERS permet d'explorer}

Fortes de ce nouvel outil que constitue le TERS, de nouvelles applications dans la caractérisation de nanomatériaux se développent et sont déjà utilisées par la communauté scientifique.

Par exemple, les liaisons que forment les atomes de carbone entre eux dans le graphène sont sensibles à la spectroscopie Raman. Le TERS permet notamment d'imager des défauts locaux dans la structure graphitique du graphène tout en corrélant cette information chimique aux propriétés physiques obtenues avec l'AFM (les variations du travail de sortie liées aux modifications du niveau de Fermi) [2]. D'autres matériaux bidimensionnels, utilisés notamment en optoélectronique pour leur gap ajustable, peuvent aussi être caractérisés par TERS comme la famille des dichalcogénures de métaux de transition (MoS2, WS2, WSe2 et bien d'autres). Le TERS permet de mettre en évidence que ce type de matériaux n'est pas homogène notamment sur ces bords concernant la densité de porteurs de charge [3]. Ces variations à l'échelle nanométrique peuvent influer sur les propriétés électriques des dispositifs utilisant ces nanomatériaux 2D. Il est aussi possible de corréler informations chimiques et informations optoélectroniques en combinant le TERS avec la TEPL (pour tip enhanced photoluminescence) reposant également sur l'exaltation et le confinement du champ par la pointe [4].

\section{Conclusion}

Le TERS permet d'accéder aux informations relatives à la spectroscopie Raman (structure chimique, cristallinité, défauts etc.) à une échelle de quelques nanomètres seulement. Cette augmentation de la résolution optique est possible grâce à l'utilisation de pointes en métal noble qui sont le siège de résonances de plasmons. Le TERS est aujourd'hui essentiellement utilisé pour caractériser les nanomatériaux (1D et 2D) et permet de faire le lien entre propriétés physiques et chimiques.

Les nouveaux développements du TERS aujourd'hui concernent les mesures in situ et notamment en milieu liquide ; ceci est en effet crucial pour étendre cette technique aux sciences de la vie. En électrochimie également, la pointe TERS pouvant être utilisée comme électrode de travail, le TERS permet de suivre des réactions d'oxydoréduction cycle par cycle et d'estimer précisément l'avancée de la réaction chimique. Cette nouvelle application ouvre ainsi la voie à l'utilisation du TERS dans le milieu de l'énergie et des batteries [5].

\section{POUR EN SAVOIR PLUS}

[1] Taguchi, A. Plasmonic tip for nano Raman microcopy: structures, materials, and enhancement, Opt. Rev. 24, 462-469 (2017)

[2] Sheremet, E., et al. Nanoscale imaging and identification of a four-component carbon sample, Carbon 96, 588-593 (2016)

[3] Kastl, C., et al. The important role of water in growth of monolayer transition metal dichalcogenides, 2D Mater. 4, 021024 (2017)

[4] Kumar, N., et al. Simultaneous topographical, electrical and optical microscopy of optoelectronic devices at the nanoscale, Nanoscale $\mathbf{9}, 2723$ (2017)

[5] Touzalin, T., et al. Complex electron transfer pathway at a microelectrode captured by in situ nanospectroscopy, Anal. Chem. 89, 8974 (2017) 